\title{
OP92
}

\section{SIMPLIFIED HYPERSPECTRAL CAMERA CALIBRATION FOR ACCURATE RADIOMETRIC MEASUREMENTS}

\author{
Aiman Raza et al.
}

DOI 10.25039/x46.2019.OP92

from

CIE x046:2019

\section{Proceedings}

of the

29th CIE SESSION

Washington D.C., USA, June 14 - 22, 2019

(DOI 10.25039/x46.2019)

The paper has been presented at the 29th CIE Session, Washington D.C., USA, June 14-22, 2019. It has not been peer-reviewed by CIE.

(C) CIE 2019

All rights reserved. Unless otherwise specified, no part of this publication may be reproduced or utilized in any form or by any means, electronic or mechanical, including photocopying and microfilm, without permission in writing from CIE Central Bureau at the address below. Any mention of organizations or products does not imply endorsement by the CIE.

This paper is made available open access for individual use. However, in all other cases all rights are reserved unless explicit permission is sought from and given by the CIE.

CIE Central Bureau

Babenbergerstrasse 9

A-1010 Vienna

Austria

Tel.: +4317143187

e-mail: ciecb@cie.co.at

www.cie.co.at 


\title{
SIMPLIFIED HYPERSPECTRAL CAMERA CALIBRATIONFOR ACCURATE RADIOMETRIC MEASUREMENTS
}

\author{
Raza, A..$^{1,2}$, Jost, S. ${ }^{1}$, Dubail M. ${ }^{2}$, Dumortier, D. ${ }^{1}$ \\ 1 University of Lyon, ENTPE, LGCB, Vaulx-en-Velin, FRANCE, \\ 2 Essilor International, Paris, FRANCE \\ razam@essilor.fr
}

DOI 10.25039/x46.2019.OP92

\begin{abstract}
Hyperspectral imaging has enhanced radiometric and colorimetric analysis of complex scenes by offering a continuous spectrum for every pixel in the entire scene. Nevertheless, hyperspectral image acquisition and spectral calibration still remain a challenging task. We propose a simplified methodology to enable accurate and reliable characterisation of a commercial hyperspectral camera and spectral calibration of its output data. A calibration coefficient was determined for the spectral acquisition range $(400 \mathrm{~nm}-1000 \mathrm{~nm})$ and native resolution (every $2,7 \mathrm{~nm}$ ) to transform the raw hyperspectral data into spectral radiance in SI units $\left(\mathrm{W} \cdot \mathrm{m}^{-2}\right.$.sr per $\mathrm{nm}$ ). This calibration curve, along-with a linear relation between exposure values and corresponding raw radiance values, was used to automatically determine exposure values that might over/under expose the camera sensors. Different spectral resolutions were also tested to minimize the data size and maintain spectral accuracy. These characterisations and the spectral calibrations were validated under LED and fluorescent sources within a $2300 \mathrm{~K}$ $6500 \mathrm{~K}$ range of correlated-colour temperatures.
\end{abstract}

Keywords: Hyperspectral imaging, Spectral calibration, Exposure setting

\section{Introduction}

In physical sciences and especially in lighting engineering, spectral analysis holds a high importance as it offers a non-invasive method to analysis object properties, ranging from their radiometric content, colorimetric appearance, reflectance properties etc. (Amani et al., 2018; Baret et al., 1987; Scotter, 1990; Shlaer et al., 1942). A spectroradiometer captures the spectral radiances of a point target within the spectral range of the acquisition system. This methodology is efficient as long as we treat simple point objects. When an entire scene has to be analysed, spectral measurement of every object in the scene is not practical owing to the time constraints and the absence of spatial features (Ariana et al., 2006). In this scenario, imaging is helpful as it allows us to analyse every object in relation to its surrounding objects, which impacts the visual perception of a certain object (Zheng et al., 2018). The major drawback of traditional imaging is the absence of detailed spectral information in narrow wavelength regions of the objects, for each pixel (point target); the only information available is in the broad wavelength (RGB) (Taghizadeh et al., 2011). This information is not sufficient in all the situations and there is an increasing need to be able to measure the spectral content of complex scenes with an acceptable accuracy.

Hyperspectral imaging provides a good solution for these concerns as it combines spectroscopy and traditional imaging offering the best of the two methodologies. Originally developed for remote sensing (Goetz et al., 1985), the current usage ranges from food quality analysis (Sun, 2010) to classification of forest tree species (Hycza et al., 2018), medical imaging (Duan et al., 2019; Gawad et al., 2019) and temperature and radiation emission studies (Zheng et al., 2019).

A hyperspectral camera captures spectral information within a particular wavelength range (UV, NIR, VIS or a mix) for each spatial target in the scene which in turn depends on the spatial resolution of the camera. A hyperspectral image has three dimensions, [RCW]; where $R$ and $C$ denote the pixel array (row and columns, e.g. $2560 \times 1440$ pixels) and $W$ denotes the wavelength dimension ( $>30$ bands generally). Current hyperspectral technology does not allow simultaneous capture of the entire spectrum for every pixel position in the scene, i.e. either the 
spectral information is stacked band by band for every pixel in the scene or multiple line by line complete spectral captures are combined to create the entire field of view. The earlier methodology of creating a hypercube involved capturing the same scene sequentially for changing wavelengths, common examples include Liquid Crystal Tuneable Filters (LCTFs) and Acousto-optic Tuneable Filters (AOTFs) (Lewis et al., 2008; Wang et al., 2018). This methodology provided a good spatial resolution and focussed images but had a smaller spectral resolution. The second approach is called 'push broom technology', where the spectral measurement is done for every point in the scene with the help of a relative motion between the camera and the scene, either a conveyer belt (moving sample) or rotary motor (moving camera) (Shi et al., 2018). These types of hyperspectral cameras are able to capture scenes with an improved spectral resolution (as high as every $1,3 \mathrm{~nm}$ ).

Despite all the benefits of hyperspectral imaging, for good quality imaging, this technology is still extremely expensive, large in size and difficult to operate for non-experts. Apart from that there are many parameters that need to be mastered for a proper hyperspectral image. The major camera parameters that can affect the result include the spatial resolution, the spectral resolution, the exposure value, the frame rate and the lens focalisation. This article will mainly focus on exposure values (which in turn depends on frame rate and luminance levels of the scene), the choice of spectral resolution and the calibration of raw radiance data of the hyper spectral camera. A hyperspectral output is usually in raw units defined by the camera manufacturer which requires calibration to produce radiance in SI units. This step is important not just for calibrating the radiance hypercube but also for characterising a typical hyperspectral output.

\section{Material and setup}

\subsection{Instruments}

A push broom hyperspectral camera named FX10 manufactured by SPECIM ${ }^{\circledR}$ was used for scene acquisition. It is composed of a CMOS sensor with a spectral resolution of $5,5 \mathrm{~nm}$ (FWHM) and a lens with an $f$ number of 1,7 . The camera operates in the visible-near infra-red region of the spectrum $(\sim 400 \mathrm{~nm}-1000 \mathrm{~nm})$. It was mounted on a rotary scanner to capture the scene.

Two spectroradiometers manufactured by $J E T I \circledR$ were used for measuring the spectral radiances and the luminance values (of the sources and the objects): the Specbos 1211UV and the Specbos 1501. A chroma-meter (Konica Minolta, CL 200A) was also placed in the centre of the scene to measure horizontal illuminance levels.

The Macbeth chart with 24 color patches was used for colorimetric measurements and a Spectralon white standard was used as the reference. In the article, the Macbeth Color Chart will be named as MCC followed by a number (to identify the color) and the Spectralon will be named as RW.

\subsection{Scene acquisition setup}

The captures were taken in a white light booth with a uniform reflectance $(\rho=0.81)$, a polycarbonate diffuser was placed at the ceiling of the light booth to uniformly distribute the light, this configuration was used for testing low to medium radiance levels. A temporary elevated block was added inside the light booth and the diffuser was removed for spectral calibration to increase the available light levels for measurements, this configuration was used for working at high radiance levels, see Figure 1. The booth was lit either by an incandescent source or by three spectrally tuneable 7-channel LED projectors (SourceFour LED, Lustr+ from the ETC Company) which were mixed to provide an equal energy spectrum (EES) and Full boosted spectrum (FS, all 7 LEDs at maximum intensity). We also had a set of four fluorescent sources though they were not inside the light booth and were used solely for validating the calibration. The spectral radiance of the three sources used for the calibration, and measured in the light booth on a reference white standard are plotted in Figure 2. 



Figure 1 - a: Low radiance level configuration; b: High radiance level configuration

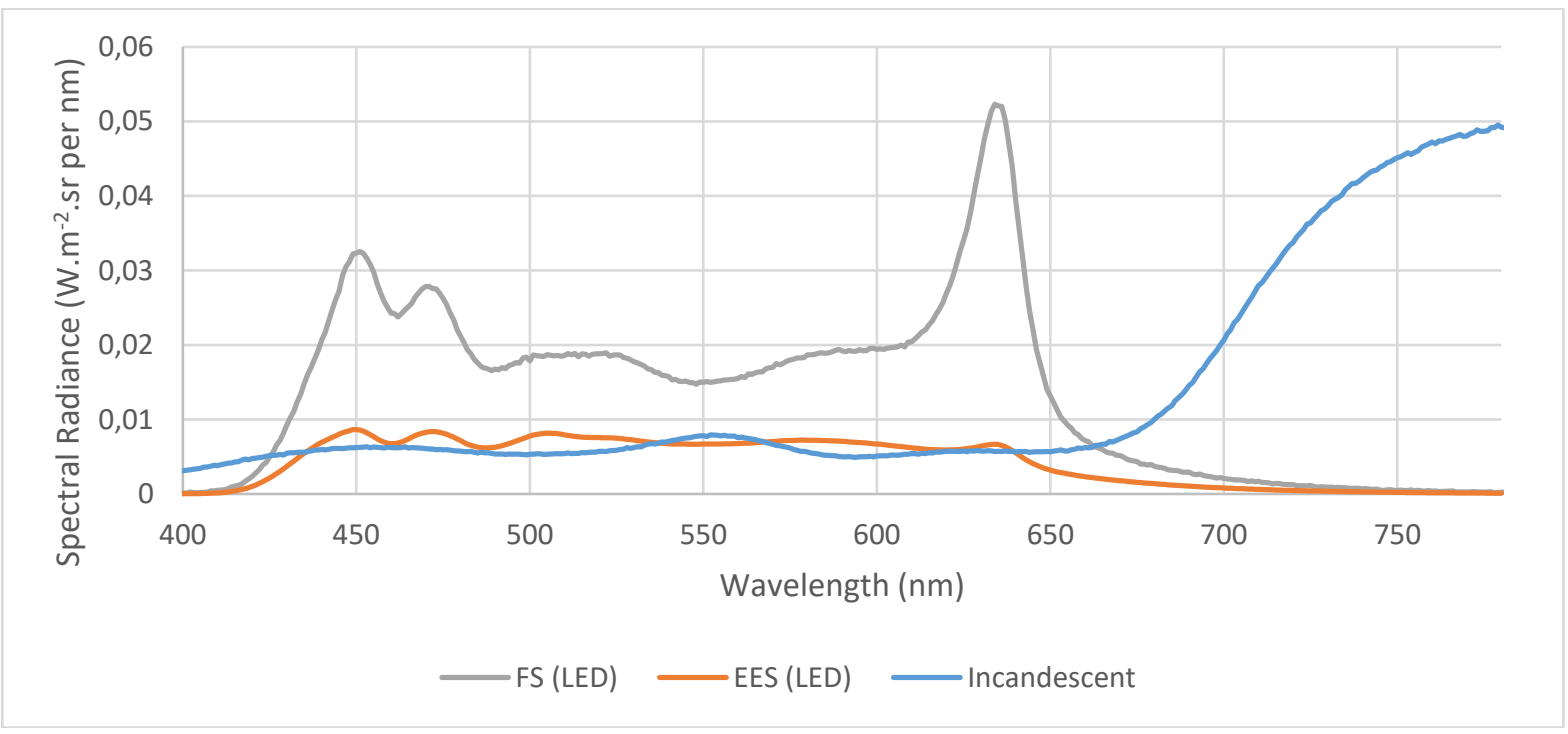

Figure 2 - Spectral radiances of light booth luminary sources

\subsection{Hyper spectral data extraction}

The spectroradiometer is equipped with a laser pointer which marks the spot being measured. Our hyperspectral camera generates a preview image of the captured scene which can be used to identify the approximate position of the spectroradiometer. A Matlab script was written using the inbuilt imellipse function to extract the hyperspectral data for the desired position in the preview image. This script was used to extract the radiance measurements for the MCC and the RW whenever needed.

\section{Spectral radiance calibration curve}

\subsection{Calibration under incandescent light}

The raw hyperspectral captures from the camera are in arbitrary units thus their spectral data do not match real radiances (the peaks and shapes are different). That is why, the raw data needs to be calibrated for spectral accuracy. To calibrate the raw radiance output of the hyperspectral camera the target scene was composed of MCC and RW illuminated with an incandescent lamp as a reference calibration source. The capture was done in the high radiance level configuration of the light booth. The spectral radiance calibration curve was determined for the entire spectral range of the camera $(\sim 400 \mathrm{~nm}-1000 \mathrm{~nm})$ and a spectral resolution of 2,7 $\mathrm{nm}$ with a fixed exposure value of $40 \mathrm{~ms}$. 
The raw radiance values obtained on the reference white with the hyperspectral image were divided with the corresponding reference spectral radiance measured with the spectroradiometer (interpolated linearly to match the spectral resolution of the hyperspectral camera). The calibration was repeated three times and the three calibration curves were averaged to finally achieve the calibration curve in Figure 3.

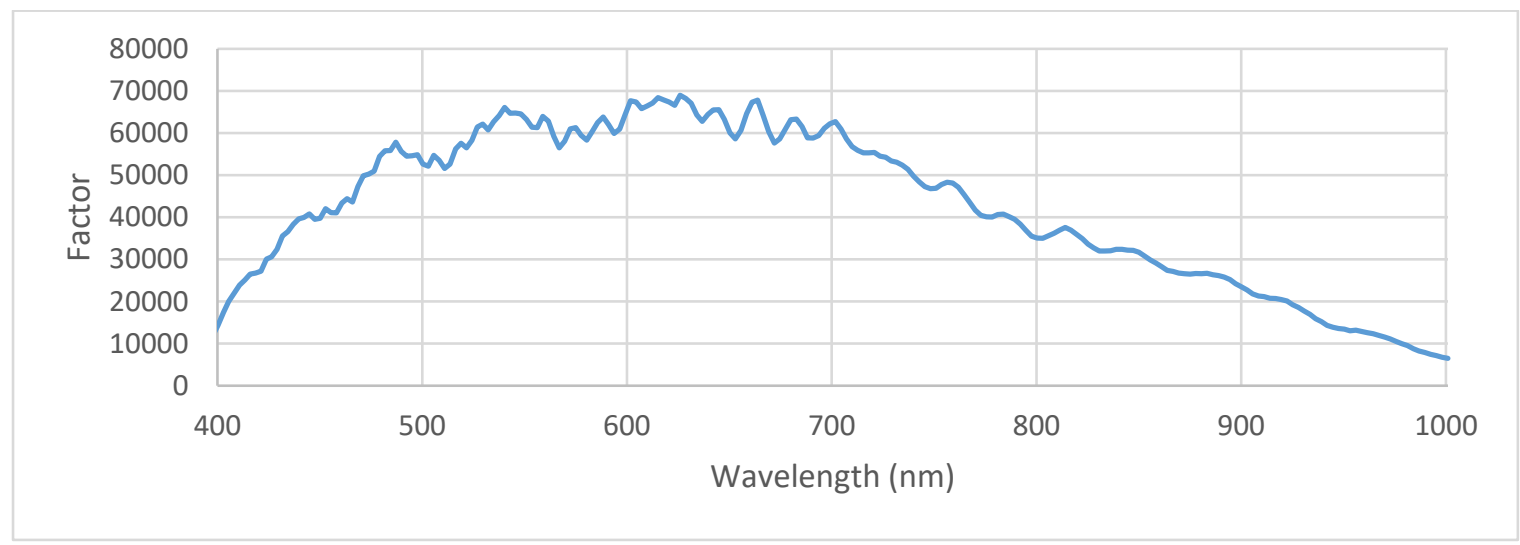

Figure 3 - Radiance calibration curve obtained with the incandescent source

\subsection{Validation}

The spectral radiance calibration curve obtained with the incandescent source was tested on EES in the high radiance level configuration of light booth with hyperspectral captures taken at $40 \mathrm{~ms}, 20 \mathrm{~ms}$ and $10 \mathrm{~ms}$ of exposure values. The spectral radiances on the RW obtained with the three captures were compared with the spectral radiance obtained from the spectroradiometer (Figure 4). The average mean square error for the three calibrated spectral radiances was $7 \times 10^{-8} \mathrm{~W} \cdot \mathrm{m}^{-2} . \mathrm{sr}$ per $\mathrm{nm}$.

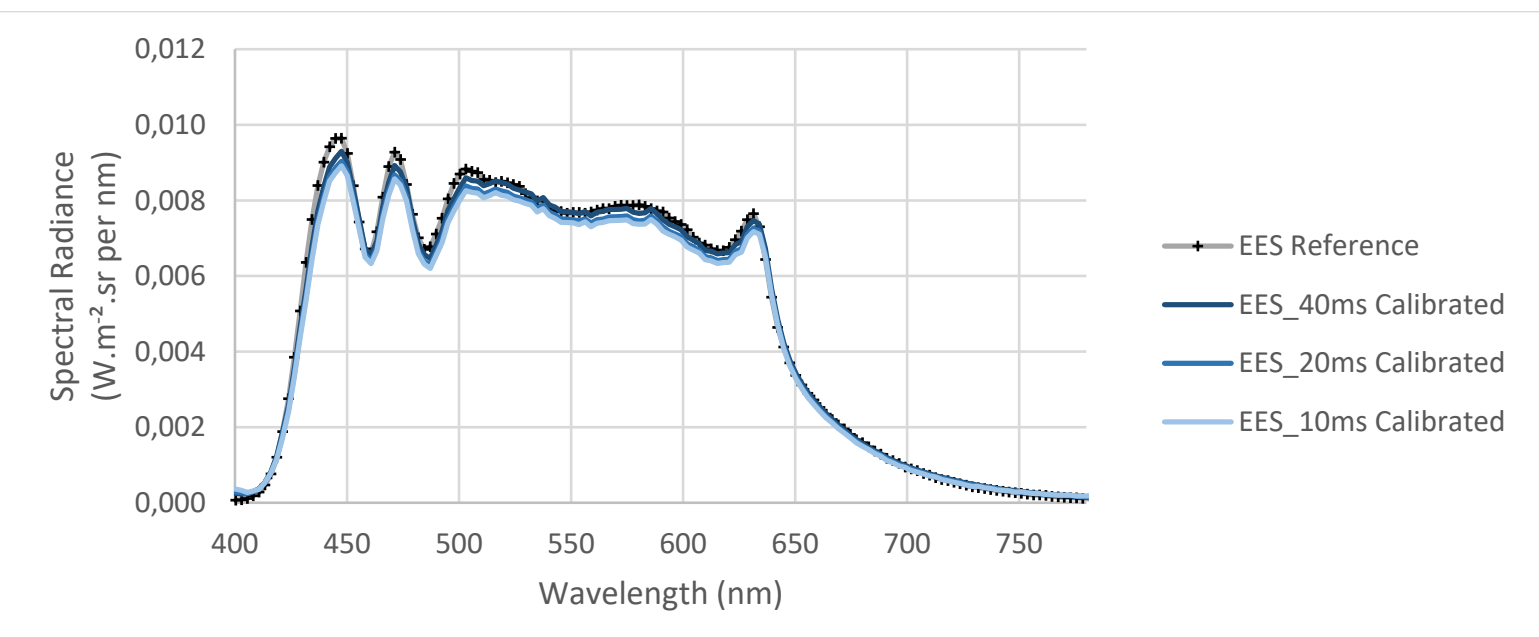

Figure 4 - Verification of spectral accuracy of the calibration curve determined under the incandescent source

This calibration curve was further verified on fourteen different sources comprising of nine LED sources and five fluorescent sources of different color temperatures ranging from $2316 \mathrm{~K}$ to $6589 \mathrm{~K}$. The Low/Medium radiance level configuration of light booth was used for all these captures where the RW and the MCC (1-24) were used as targets. The reference spectral measurements were done with the spectroradiometer for the 24 MCC samples and the RW.

The mean and maximum color difference calculated ( $\triangle E$ Eab) on the 24 color samples of MCC and on the RW are given in the Table 1. 
Table 1 - Reproduction accuracy of the calibration curve- Summary

\begin{tabular}{|c|c|c|c|c|c|c|}
\hline S.No & Lamp_type & $\mathrm{CCT}(\mathrm{K})$ & \multicolumn{2}{|c|}{$\Delta \mathrm{Eab}$} & Luminance & Illuminance \\
\hline & & & Mean & Max & On RW (cd. $\left.\mathrm{m}^{-2}\right)$ & Lux \\
\hline 1 & Fluorescent & 2895 & 5,3 & 11,3 & 160 & 910 \\
\hline 2 & Fluorescent & 3629 & 5,1 & 7,7 & 92 & 527 \\
\hline 3 & Fluorescent & 4106 & 5,2 & 8,7 & 159 & 1325 \\
\hline 4 & Fluorescent & 4749 & 5,1 & 7,2 & 97 & 558 \\
\hline 5 & Fluorescent & 5776 & 5,1 & 7,1 & 99 & 552 \\
\hline 6 & LED & 2316 & 6,9 & 15,1 & 550 & 1796 \\
\hline 7 & LED & 2535 & 3,9 & 9 & 551 & 1795 \\
\hline 8 & LED & 2704 & 4,1 & 9,9 & 544 & 1797 \\
\hline 9 & LED & 3009 & 3,6 & 9,4 & 548 & 1794 \\
\hline 10 & LED & 4009 & 2,2 & 4,8 & 551 & 1795 \\
\hline 11 & LED & 5017 & 2 & 4,3 & 553 & 1796 \\
\hline 12 & LED & 5108 & 2,7 & 5,3 & 847 & - \\
\hline 13 & LED & 5896 & 2,5 & 4,5 & 500 & - \\
\hline \multirow[t]{2}{*}{14} & \multirow[t]{2}{*}{ LED } & 6589 & 1,9 & 3,5 & \multirow[t]{2}{*}{554} & \multirow[t]{2}{*}{1795} \\
\hline & & Mean & 3,9 & 7,7 & & \\
\hline
\end{tabular}

\section{Influence of measurement parameters}

\subsection{Minimum luminance levels for hyperspectral capture}

In order to properly characterise the hyperspectral camera, it is important to understand its limitations related to luminance levels. Luminance levels which can be measured with a spectroradiometer might not be measurable by a hyperspectral camera because of a significant amount of noise. By increasing the camera exposure values, relatively cleaner hyperspectral captures are possible but with an elevated risk of noise.

To test the minimum luminance levels measurable with our camera, the Low/Medium radiance level configuration of light booth was illuminated with EES with a progressive increase in light levels (nine levels) by adjusting the intensity channel of the LED projector (measured luminance level from $0,4 \mathrm{~cd} \cdot \mathrm{m}^{-2}$ to $61,2 \mathrm{~cd} \cdot \mathrm{m}^{-2}$ on MCC 19 (top-left white square); measured illuminance levels from 3 lux to 409 lux at centre of light booth). The frame rate was set to $5 \mathrm{~Hz}$. The exposure value was set to $100 \mathrm{~ms}$. Hyperspectral captures were taken for each luminance level on the grey-white and RGBYCM Macbeth squares (MCC 13-MCC 24).

The hyperspectral captures were calibrated and then tone-mapped with the iCAM06 algorithm. The following parameters were fixed: the overall contrast, $p$ was set to 0,75 (default value for indoor scenes) and the surround factor parameter, gamma value was set to 1,5 (dim surround), (Kuang et al., 2007). The images were then visually analysed for overall image quality and amount of noise, Figure 5 (due to space constraints only eight out of nine images are shown). 


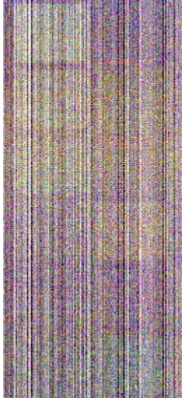

$0,4 \mathrm{~cd} \cdot \mathrm{m}^{-2}$

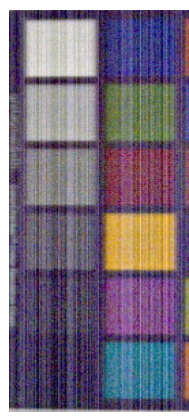

$5,4 \mathrm{~cd} \cdot \mathrm{m}^{-2}$



$13,4 \mathrm{~cd} . \mathrm{m}^{2}$

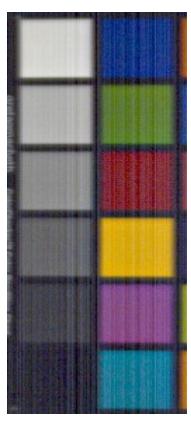

$29,4 \mathrm{~cd} \cdot \mathrm{m}^{-2} \quad 37,4 \mathrm{~cd} \cdot \mathrm{m}^{-2}$


$45,3 \mathrm{~cd} . \mathrm{m}^{-2} \quad 53,2 \mathrm{~cd} \cdot \mathrm{m}^{-2} \quad 61,2 \mathrm{~cd} \cdot \mathrm{m}^{-2}$

Figure 5 - From left to right, hyperspectral images at $100 \mathrm{~ms}$ for increasing luminance levels

As it can be seen in the figure, hyperspectral images for luminance above $50 \mathrm{~cd} \cdot \mathrm{m}^{-2}$ are visually similar with minute differences on coloured patches. Below $50 \mathrm{~cd} . \mathrm{m}^{-2}$ the noise distortions decrease with an increase in luminance.

Thus it can be assumed that hyperspectral captures with luminance conditions higher than 50 $\mathrm{cd} . \mathrm{m}^{-2}$ (on white samples) and illuminance values higher than $\sim 360$ lux can be rendered for visual reproduction but with a pre identified elevated exposure value.

\subsection{Appropriate exposure value (ms) for different luminance levels}

A spectroradiometer adjusts automatically the exposure value for each spectral capture depending on the luminance conditions, unfortunately this is not the case with hyperspectral captures. With a hyperspectral camera there is a constant risk to over or under-expose the camera lens under extreme luminance conditions (too bright or too dark). The exposure value needs to be adjusted for different luminance conditions and for a non-expert in photography this can be difficult.

To solve this problem, we developed a utility tool in MS Excel which can predict the over/under exposure of the hyperspectral camera depending on the spectral radiance of the luminary source. Hyperspectral captures were done in the high radiance level configuration of the light booth for luminance values ranging from $0 \mathrm{~cd} . \mathrm{m}^{-2}$ to $2791 \mathrm{~cd} . \mathrm{m}^{-2}$ under the EES LED source with increasing exposure values of 5-10-20-40-80 ms.

Hyperspectral captures were also done under the incandescent source for a fixed luminance level of $537 \mathrm{~cd} . \mathrm{m}^{-2}$ with exposure values changing from 1-5-10-20-30-40-50-60-70-80 ms.

These measurements helped us in identifying:

a) The exposure values which saturate the camera and are identifiable with a flat line in the EES spectral curve, thus it gave us the upper limit of the radiance values measurable by the hyperspectral camera.

b) The exposure values which under-expose the hyperspectral camera and have radiance values comparable to the noise measured for a scene with $0 \mathrm{~cd} . \mathrm{m}^{-2}$ (dark), thus it gave us the lower limit of exposure values for a particular scene.

c) A linear relation between the exposure values and corresponding raw radiance values for incandescent source, identified by an equation with $40 \mathrm{~ms}$ as the reference exposure value.

Using the linear relationship between the different exposure values and the raw radiance values from step (c) and the radiance calibration coefficient (paragraph 3.2), we were able to model a raw hyperspectral radiance curve from a spectral curve measured with a spectroradiometer. Using the upper and lower limits of the raw radiance values from steps a and b, we can identify the possible exposure values that could over/under expose the hyperspectral camera. 

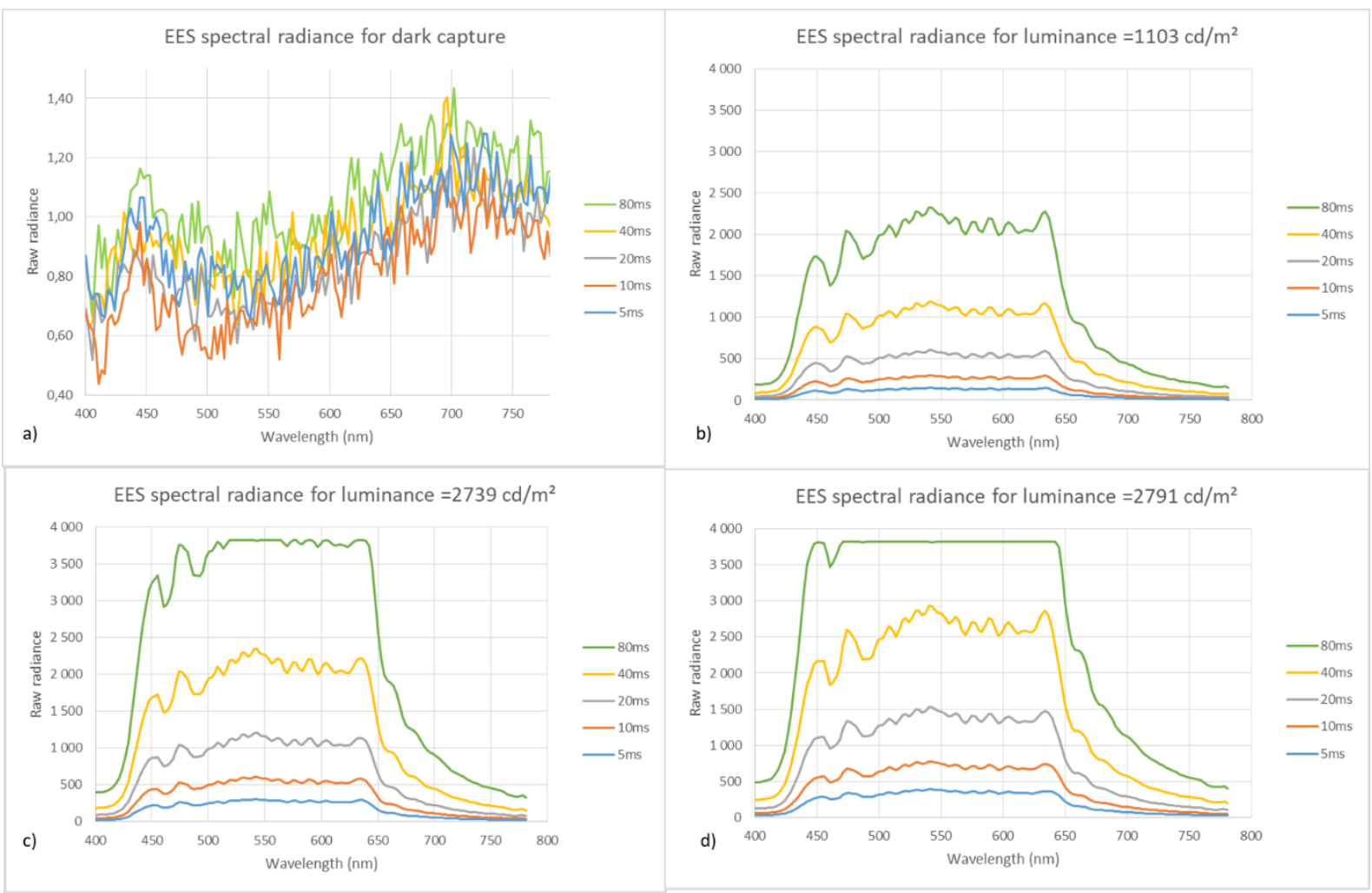

Figure 6 - Spectral radiance plots for increasing luminance levels and exposure values on Spectralon under EES LED source

As can be seen in Figure 6 (c-d), the saturation for the camera lens is clearly identifiable with a flat spectral radiance plot starting for $80 \mathrm{~ms}$ of exposure value for the luminance levels of $2739 \mathrm{~cd} . \mathrm{m}^{-2}$ and $2791 \mathrm{~cd} . \mathrm{m}^{-2}$. This saturating radiance value was identical for all over exposed exposure vales and equal to 3820 raw radiance units. To be on the safer side, the upper limit was defined as $90 \%$ of the saturated raw radiance value, thus a capture measuring raw radiance more than 3438 unit was determined as over exposed. From the spectral radiance plot of the dark capture Figure $6(\mathrm{a})$, we identified the noise measured by the camera and set a value $10 \%$ higher than the highest noise value (1,43 raw unit) as the lower limit (thus 1,58 raw unit) for a properly exposed capture.

From the hyperspectral captures under the incandescent source with a fixed luminance value of $537 \mathrm{~cd} . \mathrm{m}^{-2}$ and increasing exposure values $(1 \mathrm{~ms}-80 \mathrm{~ms})$, we identified a linear relationship between the radiance values for different exposure values while setting the radiance values from $40 \mathrm{~ms}$ of exposure as the base. These linear equations, the radiance calibration curve and the upper/lower limits of exposure values enabled us to develop a utility tool in MS Excel to automatically identify correct exposure values for the spectral radiance measured with a spectroradiometer. This tool was validated on captures done under EES, FS and Incandescent sources with exposure values of 10-20-40 ms, see Figure 7.

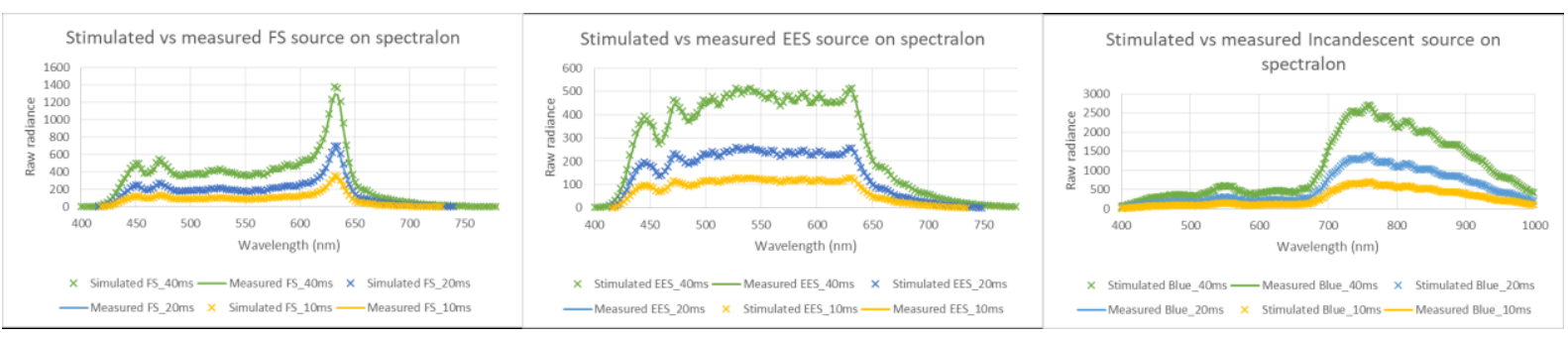

Figure 7 - Validation of automatic exposure calculation tool 


\subsection{Influence of spectral resolution on hyperspectral measurements}

Our hyperspectral camera offers different binning options which enable us to change the spectral and spatial resolution of measurements. The spatial binning was set to be native (1024 pixels) to obtain the best image quality possible. By changing the spectral resolution, it is possible to obtain hyperspectral images every $1,3 \mathrm{~nm} ; 2,7 \mathrm{~nm} ; 5,4 \mathrm{~nm}$ and $10,8 \mathrm{~nm}$. The resolution of $1,3 \mathrm{~nm}$ is in fact an interpolation of the native resolution of 2,7 $\mathrm{nm}$, thus it was not taken in consideration. The resolution of $10,8 \mathrm{~nm}$ was too low and could lead to significant loss in spectral information (Schanda, 2007) and thus was not considered as well.

To test the influence of the spectral resolution, two hyperspectral captures of MCC (1-24) and RW in the original light booth were taken for the two spectral resolutions $(2,7 \mathrm{~nm}$ and $5,4 \mathrm{~nm})$ under the FS source $\left(\mathrm{Y}=461 \mathrm{~cd} . \mathrm{m}^{-2}\right)$. With the help of Matlab, raw radiance values for the coloured samples of MCC (1-18) were extracted of the two hyperspectral images and were calibrated with the calibration coefficient.

The raw spectral data were not completely superposed for the two binning options tested $(2.7$ $\mathrm{nm}$ and 5,4 nm) for the 18 coloured patches of MCC and RW, see Figure 8 (only for RW). A detailed analysis of the obtained spectral radiances and $\Delta E a b$ as compared to the spectroradiometer measured spectral radiances for the MCC (1-18) and RW are presented in Table 2.

Table 2 - Spectral accuracy comparison for two spectral resolutions

\begin{tabular}{|l|r|r|l|}
\hline Resolution & $\begin{array}{r}\text { Mean } \\
\Delta \text { Eab }\end{array}$ & $\begin{array}{r}\text { Max } \\
\Delta \text { Eab }\end{array}$ & $\left(\right.$ W.m ${ }^{-2}$.sr per nm) \\
\hline Binning 2 & 5,1 & 8,5 & $5,24 \times 10^{-7}$ \\
\hline Binning 4 & 3,4 & 8,5 & $2,63 \times 10^{-7}$ \\
\hline
\end{tabular}

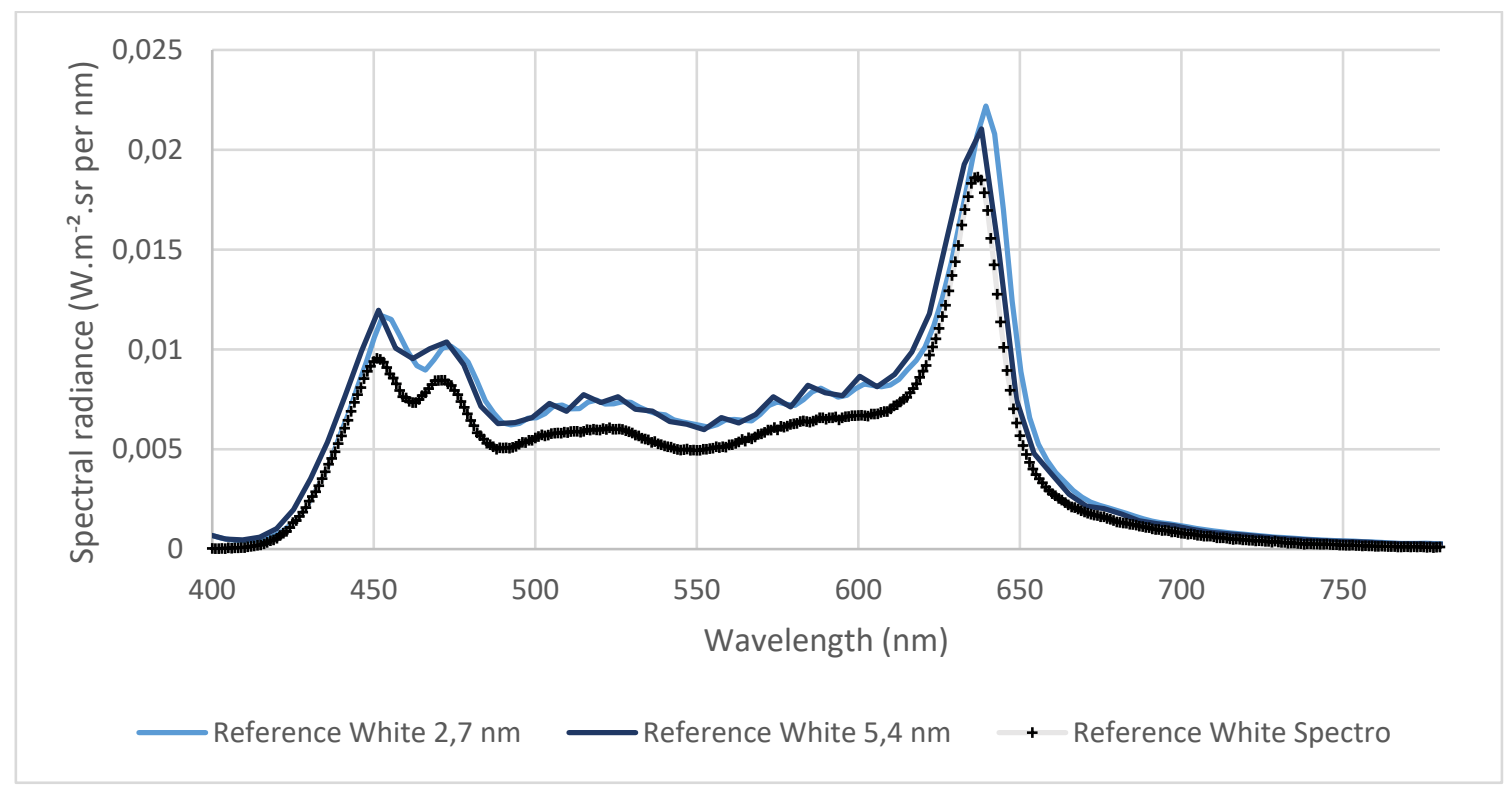

Figure 8 - Spectral binning options and effects on spectral radiance values

This analysis suggests that a spectral binning of $5,4 \mathrm{~nm}$ has an accuracy similar to a binning of $2,7 \mathrm{~nm}$ for hyperspectral captures, and since hyperspectral captures with a step size of $5,4 \mathrm{~nm}$ take half the space as compared to step size of $2,7 \mathrm{~nm}$, by using a lower resolution we can reduce data consumption. 


\section{Conclusion}

This study identified various issues associated with hyperspectral imaging and was able to provide solutions which enable proper image capture and characterisation of our hyperspectral camera. The major part of the study was related to spectral calibration of the hyperspectral data to reflect radiance values in SI units. We were able to identify a radiance factor curve for the entire spectrum range $(\sim 400 \mathrm{~nm}-1000 \mathrm{~nm})$ captured by the camera. This calibration curve when applied on other hyperspectral captures gave us a mean $\Delta$ Eab value of 3,3 ; across a range of colour temperatures, two type of sources (LED and fluorescent), 24 patches of MCC and RW. For simple colour patches, $\Delta$ Eab less than 2 is deemed to be visually indistinguishable for nonexpert observers (Mokrzycki and Tatol, 2011). It is to be noted that for a complex scene the colour perception changes in relation to the surrounding environment (Webster, 2015), chromatic adaptation (K. Shevell, 1982; Shevell and Humanski, 1988), shape and size of the colour in question (M. Stone, 2012). It has been identified that a $\Delta$ Eab less than 6 can be considered as non-perceivable difference for complex images (Hordley et al., 2004; Meyer, 1988).

We also identified that for a spectral resolution of $5,4 \mathrm{~nm}$, a hyperspectral image capture will render results with an accuracy similar to the one obtained with a resolution of $2,7 \mathrm{~nm}$. Since a capture with a resolution of $5,4 \mathrm{~nm}$ reduces the data size by half while staying close to the CIE minimum spectral resolution guidelines $(\sim 5 \mathrm{~nm})(\mathrm{CIE} 15: 2004,2004)$, the binning of 4 is a safe choice for our camera.

There are two important criteria for hyperspectral camera measurements for proper visual representation: exposure value and focussing (Shirvaikar, 2004). They are important because they cannot be easily corrected with post processing. Most of the automatic exposure calculation methods currently in use employ gradient training methods (Shim et al., 2014) or rely on multiple captures with different exposures to correct the image for a particular ROI (Nourani-Vatani, Navid and Roberts, Jonathan M, 2007). Both methods are out of scope for this study and too complicated to apply on an already manufactured hyper spectral camera. The automatic identification of exposure value proposed in this article reduces the risks of over exposed or under exposed images without modifying the camera framework or adding heavy post-processing.

\section{References}

Amani, M., Salehi, B., Mahdavi, S., Brisco, B., 2018. Spectral analysis of wetlands using multisource optical satellite imagery. ISPRS Journal of Photogrammetry and Remote Sensing 144, 119-136. https://doi.org/10.1016/j.isprsjprs.2018.07.005

Ariana, D.P., Lu, R., Guyer, D.E., 2006. Near-infrared hyperspectral reflectance imaging for detection of bruises on pickling cucumbers. Computers and Electronics in Agriculture 53, 60-70. https://doi.org/10.1016/j.compag.2006.04.001

Baret, F., Champion, I., Guyot, G., Podaire, A., 1987. Monitoring wheat canopies with a high spectral resolution radiometer. Remote Sensing of Environment 22, 367-378.

CIE 15:2004 (Ed.), 2004. Colorimetry, 3. ed. ed, Technical report / CIE. CIE, Central Bureau, Vienna.

Duan, Y., Wang, J., Hu, M., Zhou, M., Li, Q., Sun, L., Qiu, S., Wang, Y., 2019. Leukocyte classification based on spatial and spectral features of microscopic hyperspectral images. Optics \& Laser Technology 112, 530-538. https://doi.org/10.1016/j.optlastec.2018.11.057

Gawad, A.L.A., El-Sharkawy, Y., Ayoub, H.S., El-Sherif, A.F., Hassan, M.F., 2019. Classification of dental diseases using hyperspectral imaging and laser induced fluorescence. Photodiagnosis and Photodynamic Therapy 25, 128-135. https://doi.org/10.1016/j.pdpdt.2018.11.017

Goetz, A.F., Vane, G., Solomon, J.E., Rock, B.N., 1985. Imaging spectrometry for earth remote sensing. science $228,1147-1153$. 
Hordley, S.D., Finalyson, G., Morovic, P., 2004. A multi-spectral image database and its application to image rendering across illumination., in: Third International Conference on Image and Graphics, ICIG 2004, Hong Kong, China, December 18-20, 2004. pp. 394-397. https://doi.org/10.1109/ICIG.2004.10

Hycza, T., Stereńczak, K., Bałazy, R., 2018. Potential use of hyperspectral data to classify forest tree species. New Zealand Journal of Forestry Science 48. https://doi.org/10.1186/s40490-018-0123-9

K. Shevell, S., 1982. Color perception under chromatic adaptation: Equilibrium yellow and longwavelength adaptation. Vision research 22, 279-92. https://doi.org/10.1016/00426989(82)90128-6

Kuang, J., Johnson, G.M., Fairchild, M.D., 2007. iCAM06: A refined image appearance model for HDR image rendering. Journal of Visual Communication and Image Representation 18, 406-414. https://doi.org/10.1016/j.jvcir.2007.06.003

Lewis, E., Schoppelrei, J., Lee, E., Kidder, L., 2008. Near-infrared chemical imaging as a process analytical tool. Process analytical technology 187.

M. Stone, 2012. In Color Perception, Size Matters. IEEE Computer Graphics and Applications 32, 8-13. https://doi.org/10.1109/MCG.2012.37

Meyer, G.W., 1988. Wavelength selection for synthetic image generation. Computer Vision, Graphics, and Image Processing 41, 57-79. https://doi.org/10.1016/0734-189X(88)90117-X

Mokrzycki, W., Tatol, M., 2011. Color difference Delta E - A survey. Machine Graphics and Vision 20, 383-411.

Nourani-Vatani, Navid, Roberts, Jonathan M, 2007. Automatic Camera Exposure Control. Presented at the ACRA 2007, Brisbane, Australia.

Schanda, J. (Ed.), 2007. Colorimetry: understanding the CIE system. Wiley, Hoboken, NJ.

Scotter, C., 1990. Use of near infrared spectroscopy in the food industry with particular reference to its applications to on/in-line food processes. Food Control 1, 142-149. https://doi.org/10.1016/0956-7135(90)90006-X

Shevell, S.K., Humanski, R.A., 1988. Color perception under chromatic adaptation: Red/green equilibria with adapted short-wavelength-sensitive cones. Vision Research 28, 1345-1356. https://doi.org/10.1016/0042-6989(88)90066-1

Shi, S., Gong, X., Mu, Y., Finch, K., Gamez, G., 2018. Geometric super-resolution on pushbroom hyperspectral imaging for plasma optical emission spectroscopy. J. Anal. At. Spectrom. 33, 1745-1752. https://doi.org/10.1039/C8JA00235E

Shim, I., Lee, J.-Y., Kweon, I.S., 2014. Auto-adjusting camera exposure for outdoor robotics using gradient information, in: 2014 IEEE/RSJ International Conference on Intelligent Robots and Systems. Presented at the 2014 IEEE/RSJ International Conference on Intelligent Robots and Systems (IROS 2014), IEEE, Chicago, IL, USA, pp. 1011-1017. https://doi.org/10.1109/IROS.2014.6942682

Shirvaikar, M.V., 2004. An optimal measure for camera focus and exposure, in: Thirty-Sixth Southeastern Symposium on System Theory, 2004. Proceedings of The. Presented at the Thirty-Sixth Southeastern Symposium on System Theory, 2004., IEEE, Atlanta, GA, USA, pp. 472-475. https://doi.org/10.1109/SSST.2004.1295702

Shlaer, S., Smith, E.L., Chase, A.M., 1942. VISUAL ACUITY AND ILLUMINATION IN DIFFERENT SPECTRAL REGIONS. J Gen Physiol 25, 553. https://doi.org/10.1085/jgp.25.4.553

Sun, D.-W. (Ed.), 2010. Hyperspectral imaging for food quality analysis and control, 1. ed. ed. Academic, London.

Taghizadeh, M., Gowen, A.A., O'Donnell, C.P., 2011. Comparison of hyperspectral imaging with conventional RGB imaging for quality evaluation of Agaricus bisporus mushrooms. Biosystems Engineering 108, 191-194. https://doi.org/10.1016/j.biosystemseng.2010.10.005 
Wang, X., Zhang, Y., Ma, X., Xu, T., Arce, G.R., 2018. Compressive spectral imaging system based on liquid crystal tunable filter. Opt. Express 26, 25226-25243. https://doi.org/10.1364/OE.26.025226

Webster, M., 2015. Environmental Influences on Color Vision, in: Luo, R. (Ed.), Encyclopedia of Color Science and Technology. Springer Berlin Heidelberg, Berlin, Heidelberg, pp. 1-6. https://doi.org/10.1007/978-3-642-27851-8_76-3

Zheng, H., Cheng, T., Li, D., Zhou, X., Yao, X., Tian, Y., Cao, W., Zhu, Y., 2018. Evaluation of RGB, Color-Infrared and Multispectral Images Acquired from Unmanned Aerial Systems for the Estimation of Nitrogen Accumulation in Rice. Remote Sensing 10, 824. https://doi.org/10.3390/rs10060824

Zheng, S., Ni, L., Liu, H., Zhou, H., 2019. Measurement of the distribution of temperature and emissivity of a candle flame using hyperspectral imaging technique. Optik 183, 222-231. https://doi.org/10.1016/j.ijleo.2019.02.077 